\title{
Metal-insulator transition and superconductivity in highly boron-doped nanocrystalline diamond films
}

\author{
P. Achatz ${ }^{*}, 1,2,3$, E. Bustarret ${ }^{1}$, C. Marcenat $^{2}$, R. Piquerel ${ }^{2}$, T. Dubouchet ${ }^{2}$, C. Chapelier ${ }^{2}$, A.M. Bonnot ${ }^{1}$, O.A. \\ Williams $^{4}$, K. Haenen ${ }^{4}$, W. Gajewski ${ }^{3}$, J.A. Garrido ${ }^{3}$, M. Stutzmann ${ }^{3}$ \\ ${ }^{1}$ Institut Néel, CNRS et Université Joseph Fourier, B. P. 166, 38042 Grenoble Cedex 9, France \\ ${ }^{2}$ CEA, INAC, SPSMS, LaTEQS, F-38054 Grenoble Cedex 9, France \\ ${ }^{3}$ Walter Schottky Institut, Technische Universität München, Am Coulombwall, D-85748 Garching, Germany \\ ${ }^{4}$ IMO, Hasselt University, Belgium, and Division IMOMEC, IMEC vzw, Belgium
}

Received XXXX, revised XXXX, accepted XXXX

Published online XXXX

PACS

${ }^{*}$ Corresponding author: e-mail philipp.achatz@grenoble.cnrs.fr, Phone: +xx-xx-xxxxxxx, Fax: +xx-xx-xxx

The low temperature electronic transport of highly boron-doped nanocrystalline diamond films is studied down to $300 \mathrm{mK}$. The films show superconducting properties with critical temperatures $T_{c}$ up to $2.1 \mathrm{~K}$. The metal-insulator transition and superconductivity is driven by the dopant concentration and greatly influenced by the granularity in this system, as compared to highly boron-doped single crystal diamond. The critical boron concentration for the metal-insulator transition lies in the range from $2.3 \times 10^{20} \mathrm{~cm}^{-3}$ up to $2.9 \times 10^{20} \mathrm{~cm}^{-3}$, as determined from transport measurements at low temperatures. Insulating nanocrystalline samples follow an Efros-Shklovskii type of temperature dependence for the conductivity up to room temperature, in contrast to Mott variable range hopping in the case of insulating single crystal diamond close to the metalinsulator transition.
The electronic transport in the metallic samples not only depends on the properties of the grains (highly borondoped single crystal diamond) alone, but also on the intergranular coupling between the grains. The Josephson coupling between the grains plays an important role for the superconductivity in this system, leading to a superconducting transition with global phase coherence at sufficiently low temperatures. Metallic nanocrystalline samples show similarities to highly boron-doped single crystal diamond. However, metallic samples close to the metal-insulator transition show a more rich behaviour. A peak in the low-temperature magnetoresistance measurements for samples close to the transition is explained due to corrections to the conductance according to superconducting fluctuations.
1 Introduction Renewed interest in doping-induced electronic phase transitions came up as superconductivity was discovered in heavily boron-doped polycrystalline diamond prepared in a high pressure high temperature process [1], and was confirmed by several other groups [2] [3] [4] [5] [6] [7] in polycrystalline, single crystalline, and nanocrystalline boron-doped diamond (see Ref. 8 and 9, and references therein). Most experimental and theoretical work suggest a standard BCS approach involving a phonon-mediated pairing mechanism (see Ref. 8 and 9, and references therein), in contrast to more exotic descriptions based on the resonating valence band model [10] [11] or using weak localization as a precursor for superconductivity [12] [13] [14]. In the latter case, a spin-flip-driven pairing of holes weakly localized in the vicinity of the Fermi level accounts for the superconductivity. The pairing mechanism is therefore a still open question, as recently discussed [15]. 
However, in this paper we focus on the metal-insulator transition in highly boron-doped nanocrystalline diamond (B:ncd) thin films, clearly showing the influence of the dopant concentration and of the granular structure on the transport properties at low temperatures. A comparison is presented with respect to highly boron-doped single crystalline diamond [17]. Tuning the microscopic parameters, a granular metallic material can vary from a relatively good metal to an insulator, depending on the strength of electron tunneling coupling between conducting grains. Superconductivity is confirmed to occur at low temperatures in metallic nanocrystalline diamond.

2 Experimental Nanocrystalline diamond films have been prepared using microwave plasma-enhanced chemical vapor deposition (MPCVD) from a hydrogen-rich gas phase, with $\mathrm{CH}_{4} / \mathrm{H}_{2} \leq 4 \%$. Boron-doping was achieved by adding trimethylboron to the gas phase with molar concentrations $B / C$ reaching $10000 \mathrm{ppm}$ (see Tab. 1). The quartz substrate (suitable for transport measurements) were either prepared by mechanical polishing (Bmec prefix) or by ultrasonic seeding (Bus prefix) with a monodisperse solution of nanodiamond particles, in order to provide a high density of nucleation sites for film growth [18]. The substrate temperature was maintained at $700{ }^{\circ} \mathrm{C}$, while the total pressure and input power were varied between 30 to 40 Torr and 2500 to $3500 \mathrm{~W}$, respectively. The typical sample thickness is about $200 \mathrm{~nm}$, with grain sizes of about 150 nm.

A van der Pauw geometry has been used for the measurements of resistivities and Hall coefficients. Care was taken to measure in the ohmic range. The Hall effect was measured at room temperature switching the magnetic field between $+1.8 \mathrm{~T}$ and $-1.8 \mathrm{~T}$. From room temperature down to $360 \mathrm{mK}$, electrical resistivity measurements were performed using a Quantum Design Physical Properties Measurements System. A four terminal configuration for the contacts has been used. In the same setup, magnetoresistance measurements were performed with perpendicular fields up to $8 \mathrm{~T}$. A dilution fridge was used at lower temperatures with perpendicular fields up to $14 \mathrm{~T}$. Micro-Raman backscattering studies have been performed at room temperature using a $\mathrm{HeCd} \mathrm{cw}$ laser $(325 \mathrm{~nm})$ and a x $40 \mathrm{objec}-$ tive, with a resolution of about $5 \mathrm{~cm}^{-1}$. A liquid nitrogen cooled CCD was used for signal collection. Atomic Force Microscopy has been used in tapping mode with a tip consisting of a carbon nanotube. The boron concentration $n_{B}$ has been deduced from Secondary Ion Mass Spectroscopy (SIMS) depth profiles of ${ }^{11} B^{-},{ }^{12} C^{-}$, and ${ }^{11} B^{12} C^{-}$ions.

3 Results and Discussion The UV-Raman spectra shown in Fig. 1 display the presence of the $D$ and $G$ peaks [19], originating from $s p^{2}$-bonded carbon in the grain boundaries, as well as the central phonon line at $1332 \mathrm{~cm}^{-1}$ of diamond. With increasing doping a shift of the central phonon line to lower energies and a clear Fano

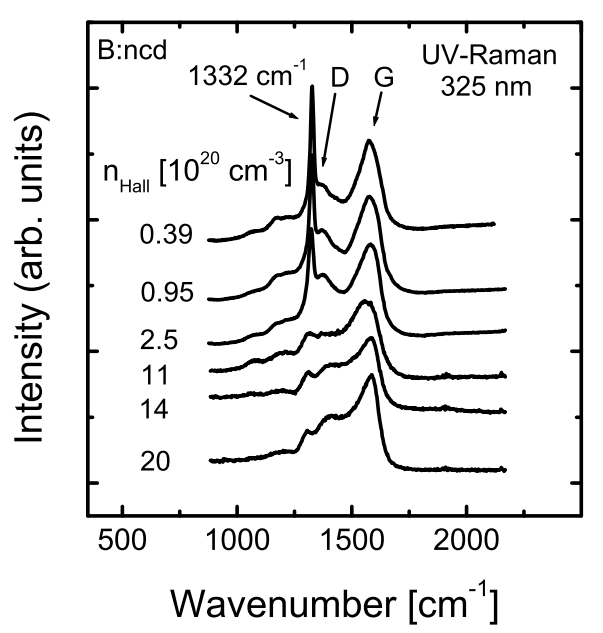

Figure 1 UV-Raman spectra $(325 \mathrm{~nm})$ display the central phonon line at $1332 \mathrm{~cm}^{-1}$ of diamond, shifting to lower energies with increasing boron doping. A clear Fano resonance can be seen. In addition, the $\mathrm{D}$ and $\mathrm{G}$ peaks, originating from $s p^{2}$ bonded carbon in the grain boundaries are also visible.

resonance can be seen, as already reported in highly borondoped single crystalline, polycrystalline, and nanocrystalline diamond [7] [20] [21] [22] [23].

Fig. 2 shows a typical AFM image $(0.5 \mu m \times 1 \mu m)$ of a boron-doped nanocrystalline diamond sample. The images have been taken using a carbon nanotube as AFM tip. Note that the topological structure measured in height is not as detailed as the information obtained from the phase image. The typical sample thickness is about $200 \mathrm{~nm}$, with grain sizes about $150 \mathrm{~nm}$. The grain size does not depend on the dopant concentration [24].

Fig. 3 shows the room temperature conductivity as a function of the free carrier concentration as determined from room temperature Hall effect measurements for several boron-doped nanocrystalline diamond thin films with boron concentrations (SIMS) in the range from $9.7 \times 10^{16} \mathrm{~cm}^{-3}$ up to $3.0 \times 10^{21} \mathrm{~cm}^{-3}$ (see Tab. 1 ). A strong increase of almost 10 orders of magnitude from $10^{-8}(\Omega \mathrm{cm})^{-1}$ up to $75(\Omega \mathrm{cm})^{-1}$ already indicates a transition from insulating to metallic samples. Compared to the room temperature conductivity of heavily boron-doped single crystal diamond, boron-doped nanocrystalline diamond films have substantially lower conductivities in the high doping range (one order of magnitude) [17] [23] . High temperature transport measurements on weaklydoped nanocrystalline diamond films reveal an activation energy of about $390 \mathrm{meV}$ [24], and, thus, indicate substitutional incorporation of boron in these films (see Ref. 18 and references therein). Assuming full ionization at very high doping levels, the difference between the Hall carrier concentration and the boron concentration as determined 
a)

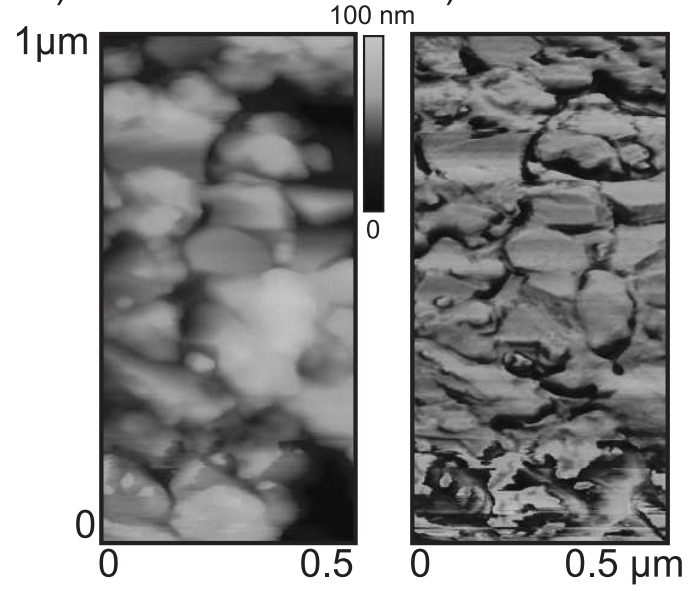

Figure 2 Typical AFM image taken with a carbon nanotube tip of a nanocrystalline diamond sample with grain size of about 100 $\mathrm{nm}$. Note that the topological structure is better revealed in (b) the phase rather than (a) the height.

\begin{tabular}{|l||r|r|r|}
\hline Sample $[$ TMB] & $n_{\text {Hall,300K }}$ & {$[B]$} & $\sigma_{300 K}$ \\
\hline ppm & {$\left[\mathrm{cm}^{-3}\right]$} & {$\left[\mathrm{cm}^{-3}\right]$} & {$\left[\Omega^{-1} \mathrm{~cm}^{-1}\right]$} \\
\hline \hline Bus0 & $<10^{15}$ & $9.7 \cdot 10^{16}$ & $1.5 \cdot 10^{-8}$ \\
\hline Bmec50 & $1.2 \cdot 10^{16}$ & - & $3.4 \cdot 10^{-3}$ \\
\hline Bmec150 & $7.0 \cdot 10^{15}$ & $2.4 \cdot 10^{19}$ & $2.4 \cdot 10^{-3}$ \\
\hline Bus20 & $7.0 \cdot 10^{18}$ & - & 0.25 \\
\hline Bus100 & $9.5 \cdot 10^{19}$ & - & 0.30 \\
\hline Bmec2500 & $3.9 \cdot 10^{19}$ & $2.3 \cdot 10^{20}$ & 0.8 \\
\hline Bus1000 & $2.5 \cdot 10^{20}$ & $2.9 \cdot 10^{20}$ & 6.2 \\
\hline Bmec5000 & $5.6 \cdot 10^{20}$ & $1.3 \cdot 10^{21}$ & 21.7 \\
\hline Bus3000 & $1.1 \cdot 10^{21}$ & $1.3 \cdot 10^{21}$ & 29.3 \\
\hline Bus4000 & & $3.0 \cdot 10^{21}$ & 34.9 \\
\hline Bus4500 & $1.1 \cdot 10^{21}$ & $1.8 \cdot 10^{21}$ & 63.3 \\
\hline Bus10000 & $1.4 \cdot 10^{21}$ & - & 55.2 \\
\hline Bus6660 & $2.0 \cdot 10^{21}$ & $2.7 \cdot 10^{21}$ & 75.8 \\
\hline Bus5000 & $2.4 \cdot 10^{21}$ & - & 72.2 \\
\hline
\end{tabular}

Table 1 The samples are designated after the seeding procedure on the substrate before growth and the boron concentration in the gas phase during growth in ppm. The carrier concentration and the conductivity as determined from Hall and van der Pauw measurements taken at room temperature, as well as the results of SIMS measurements are summarized. Sample Bus0 showed no detectable Hall effect.

by SIMS (see Tab. 1) indicates the compensating effects of the grain boundaries. For heavily boron-doped single crystal diamond, the Hall carrier concentration gives values up to ten times higher than the boron concentration measured by SIMS [17] which is not yet fully understood.

The temperature dependence of the normalized resistance $\rho / \rho(300 K)$ in the range $300 \mathrm{~K}$ down to 350

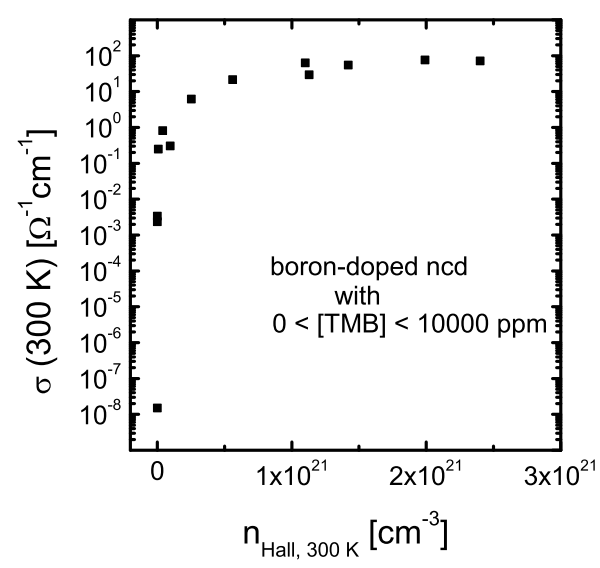

Figure 3 Room temperature conductivity as a function of the free carrier concentration, as determined from room temperature Hall effect measurements, shown for several boron-doped nanocrystalline diamond thin films with boron concentrations (SIMS) in the range from $9.710^{16} \mathrm{~cm}^{-3}$ up to $3.010^{21} \mathrm{~cm}^{-3}$ (see Tab. 1). The strong increase of the conductivity with boron doping, from $10^{-8}(\Omega \mathrm{cm})^{-1}$ up to $75(\Omega \mathrm{cm})^{-1}$, already indicates the transition from the insulating to the metallic samples.

$\mathrm{mK}$ is shown in Fig. 4 (a) for several samples. In contrast to the minimum resistivity observed in single crystal diamond at intermediate temperatures [17], the resistivity of nanocrystalline diamond increases with decreasing temperature [24]. The highly doped samples with $n_{B} \geq 1.3 \times 10^{21} \mathrm{~cm}^{-3}$ (see Tab. 1) also show superconductivity with a critical temperatures of about $T_{c}=2.1 \mathrm{~K}$ (Samples Bus1000 and Bme5000 behaved like a disordered metal, but showed no superconductivity down to $350 \mathrm{mK}$ ). Fig. 4 (b) shows a zoom of the normalized resistance $R / R(300 K)$ for sample Bus5000, compared to the diamagnetic response obtained from a.c. susceptibility measurements. The onset of diamagnetic screening corresponds to the transition to zero resistance, clearly indicating the bulk nature of superconductivity in highly boron-doped nanocrystalline diamond. The critical boron concentration $n_{c}$ for the metal-insulator transition lies in the range from $2.3 \times 10^{20} \mathrm{~cm}^{-3}$ up to $2.9 \times 10^{20} \mathrm{~cm}^{-3}$, in good agreement with what was found for single crystal and polycrystalline diamond (see Ref. 8 and 9, and references therein). As will be shown below, the metal-insulator transition is due to the increasing doping within the grains. For metallic samples close to the transition, the granular nature is becoming important, reflected by the intergranular coupling between metallic grains.

Fig. 5 (a) shows the temperature dependence of the normalized conductivity for the weakly-doped samples Bus100 and Bmec2500, which are close to the metalinsulator transition. A Efros-Shklovskii (ES) type of hopping dependence is observed over a remarkably wide range 
a)

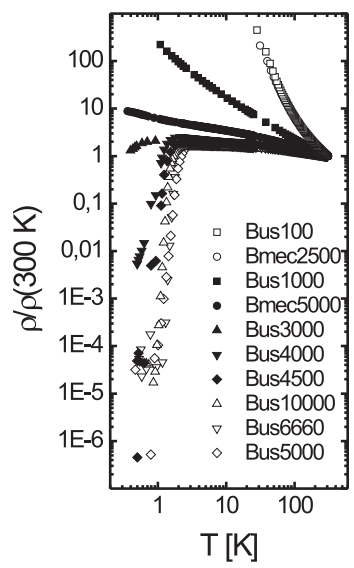

b)

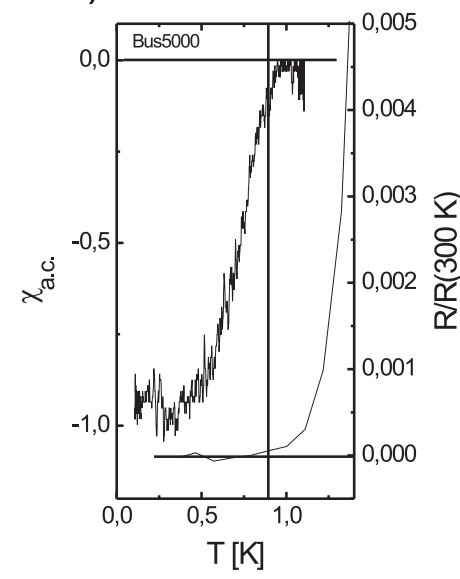

a)

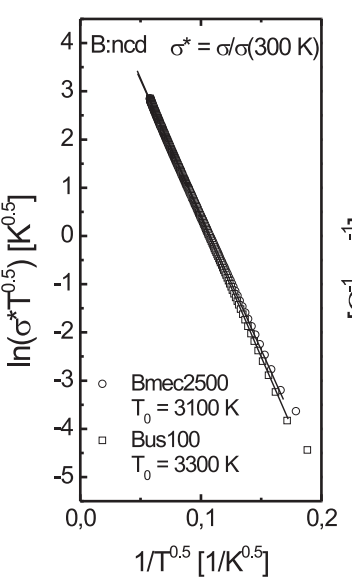

b)

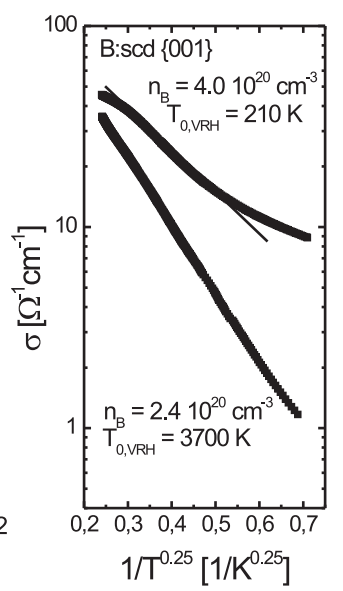

Figure 4 (a) Temperature dependence of the normalized resistance $\rho / \rho(300 K)$ from $300 \mathrm{~K}$ down to $350 \mathrm{mK}$. Highly doped samples with $n_{B} \geq 1.310^{21} \mathrm{~cm}^{-3}$ show superconductivity with a critical temperatures of about $T_{c}=2.1 \mathrm{~K}$; (b) Zoom of the normalized resistance $R / R(300 K)$ for sample Bus5000, compared to the diamagnetic response obtained from a.c. susceptibility measurements. The onset of diamagnetic screening corresponds to the transition to zero resistance.

of temperatures, from $300 \mathrm{~K}$ down to $20 \mathrm{~K}$. The exponential decrease of conductivity with decreasing temperature is typically seen on the insulating side of disordered materials such as heavily doped semiconductors. The interplay between thermal activation and tunneling processes leads to hopping transport [25] [26], characterized by a stretched exponential temperature dependence of the conductivity following

$$
\sigma(T)=\sigma_{0} \exp \left[-\left(\frac{T_{0}}{T}\right)^{m}\right]
$$

with $\mathrm{m}$ depending on the hopping mechanism. For nearestneighbour hopping (NNH) m equals unity for simple activated behaviour [27], whereas $m=1 / 4$ in the case of variable range hopping (VRH), as predicted by Mott [28]. Taking into account that Coulomb interaction leads to a reduction of the single-particle density of states near the Fermi energy and, thus, to an opening up of the so-called Coulomb gap, the hopping mechanism is called EfrosShklovskii VRH with $m=1 / 2$ instead of $m=1 / 4$ [29] [30] [31]. The Coulomb interaction plays an important role at low temperatures, and the crossover from Mott VRH to ES-hopping should be observed below a certain critical temperature. As shown in Fig. 5 (b) for highly borondoped single crystal diamond, insulating samples close to the metal-insulator transition exhibit Mott VRH and no crossover to Efros-Shklovskii VRH could be seen, probably due to the decrease of the $T_{0}$ value when approaching the metal-insulator transition. A crossover from Mott
Figure 5 (a) Temperature dependence of the normalized conductivity for the weakly doped samples Bus100 and Bmec2500, following a Efros-Shklovskii hopping over a wide range of temperatures. The $T_{0}$ values of both samples are indicated; (b) The temperature dependence of the conductivity of highly boron-doped single crystal diamond follows a Mott variable range hopping dependence.

VRH to Efros-Shklovskii VRH has recently been reported by Tshepe et al. in boron-ion-implanted films [16] as well as by Sato et al. in a lower-doped single crystal diamond [32]. The theoretical value of $T_{0, E S}$ for Efros-Shklovskii VRH in Eq. 1 is given by

$$
T_{0, E S}=\frac{e^{2} \beta}{4 \pi k_{B} \epsilon_{0} \epsilon_{r} \xi}
$$

with $\epsilon_{r}$ the relative permittivity and $\xi$ the localization length (decay length of wave function for isolated impurity atoms). The coefficient $\beta$ is equal to 2.8 according to Shklovskii [26] or equal to 0.57 according to Adkins [33]. Taking $\epsilon_{r}=5.7, \beta=2.8$, and $\xi=0.3 \mathrm{~nm}$ as the effective Bohr radius of the boron acceptor in diamond [34], one obtains for $n \ll n_{c} T_{0, E S}=2.7 \times 10^{4} \mathrm{~K}$. From the fit of the low temperature transport data of the low doped samples Bus100 and Bmec2500 we find $T_{0, E S}$ values of the order of $3000 \mathrm{~K}$ (see Fig. 5 (a)), which is a factor of 9 smaller than the theoretically predicted value. A discrepancy of a factor 5 can be explained by the choice of the value $\beta$. Another possibility is the scaling of $T_{0, E S}$ when approaching the metal-insulator transition[16], or a temperature-induced smearing of the Coulomb gap [35] [36].

An Efros-Shklovskii hopping conductivity over a wide range of temperatures has also been found in metallic and semiconducting granular arrays [37] [38] [39] [40] [41] [42] [43]. Multiple cotunneling processes can account for Efros-Shklovskii-like variable range hopping in granular 
metal arrays with weak intergrain coupling [43] [44] [45] [46]. For quantum dot arrays, random charging of dots and long range Coulomb interactions create a soft Coulomb gap [47].

A granular material is characterized by several physical quantities: the dimensionless intragranular conductance $g_{0}$, the intergranular (tunneling) conductance $g^{1}$, the charging energy $E_{c}=e^{2} / 4 \pi k_{B} \epsilon_{0} \epsilon_{r} d$ ( $\mathrm{d}$ is the grain size), and the mean level spacing $\delta=(\nu V)^{-1}$ ( $\nu$ is the density of states and $\mathrm{V}$ the volume of the grain) [44]. In the limit $g \ll g_{0}$ one obtaines a "granular system", whereas the case $g \sim g_{0}$ can be interpreted as a homogeneously disordered system. Metallic transport is expected in samples with $g>g_{c}$, whereas samples with $g<g_{c}$ show insulating behaviour. This corresponds to a situation with metallic grains, either with strong or weak intergrain coupling, respectively. The critical conductance $g_{c}$ is related to $E_{c}$ and $\delta$ as

$$
g_{c}=(1 / 2 \pi D) \ln \left(E_{c} / \delta\right)
$$

where $\mathrm{D}$ is the dimensionality [44]. For a grain size $d \approx 150 \mathrm{~nm}$ and a typical density of states $\nu \approx$ $10^{20} \mathrm{eV}^{-1} \mathrm{~cm}^{-3}$ one gets $E_{c} \approx 20 \mathrm{~K}$ and $\delta \approx 1 \mathrm{mK}$. One could expect two-dimensional effects, as the grain size is of the same order of magnitude as the thickness and, thus, $g_{c} \approx 0.52$ or $g_{c} \approx 0.77$ for a three-dimensional or two-dimensional system. These estimates lie between the values of conductances for samples Bus100 and Bus1000 (see Tab. 1). This is in good agreement with the criterion of effective activation energy used above to determine the metal-insulator transition in this material [24]. One more energy parameter has to be added if the constituent grains are made of a superconductor material: the superconducting gap $\Delta$ of the material of a single grain. Global macroscopic superconductivity can be obtained, depending on the interplay between Josephson coupling $J$ and grain charging Coulomb energy $E_{c}$.

As already mentioned above, for $g<g_{c}$ insulating behaviour is predicted following an Efros-Shklovskii variable range hopping, which is observed in nanocrystalline diamond over a wide temperature range (see Fig. 5 (a)). An estimate of the localization length $\xi$ from the experimentally determined $T_{0}$ values gives $\xi \approx 1 \mathrm{~nm}$, which is much smaller than the typical grain size. Therefore, an interpretation as granular metallic system with weak intergranular coupling, leading to an insulating behaviour, is not applicable in our case. However, as will be shown below, the granular character plays an important role for metallic nanocrystalline diamond samples close to the metal-insulator transition. The well-controlled growth of nanocrystalline diamond opens a way to study the above mentioned insulating behaviour of a metallic granular system. The doping concentration controls the intragranular

\footnotetext{
${ }^{1}$ Both $g_{0}$ and $g$ dimensionless conductance (corresponding to one spin component) and measured in the units of the quantum conductance $e^{2} / \hbar: g=G /\left(2 e^{2} / \hbar\right)$ with $\mathrm{G}$ the conductance $\left([G]=\Omega^{-1}\right)$.
}

a)

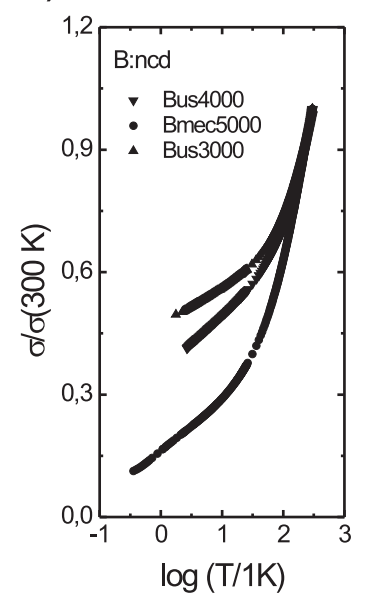

b)

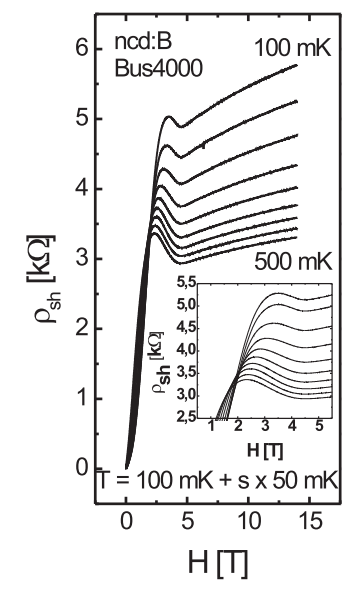

Figure 6 (a) Logarithmic temperature dependence of the normalized conductivity for several highly doped samples as expected for a granular metal ( $T$ in units of Kelvin); (b) Magnetoresistance of sample Bus4000 at several constant temperatures, revealing regions with negative and positive magnetoresistance, respectively. Inset shows a zoom in of the same graph.

conductance, whereas other growth parameters (e.g. nucleation density) determine the intergranular coupling.

For samples with $g>g_{c}$, several contributions to the conductivity can be seen, depending on the temperature range (see Ref. 42 and references therein). For temperatures $T>\Gamma(\Gamma=g \delta$ can be interpreted as the width of the smearing of the energy levels in the grains), one should find a logarithmic temperature dependence (for two- as well as three-dimensional systems) of the conductivity[44]:

$$
\sigma_{T}=\sigma_{0}\left(1-\frac{1}{\pi z g}\right) \ln \left[\frac{g E_{c}}{T}\right]=a+b \ln (T)
$$

with $\mathrm{z}$ the coordination number of the arbitrary periodic lattice ( $E_{c}$ taken in Kelvin, or $T$ in units of Kelvin).

This logarithmic temperature dependence of the conductivity has been reported for several granular metallic systems [39] [48] [49] [50] [51]. As can be seen in Fig. 6 (a), some of the nanocrystalline samples show a logarithmic temperature dependence at low temperatures. Highly conducting samples show a crossover to superconducting behaviour (not shown in Fig. 6 (a)). The temperature at which the deviation from the logarithmic temperature dependence takes place increases with doping. Electronelectron interaction is responsible for the logarithmic temperature dependence. Interactions with phonons leads to a power law dependence at higher temperatures. As discussed below, no clear logarithmic temperature dependence can be seen for more highly doped samples.

Fig. 6 (b) shows the magnetoresistance of sample Bus4000 at several constant low temperatures in the range 
from 100 up to $500 \mathrm{mK}$ and with perpendicular fields up to $14 \mathrm{~T}$. Extremely strong magnetic fields destroy the bulk superconductivity (superconductivity of single grains). The positive magnetoresistance in the high-field limit resembles the behaviour seen in metallic single crystal and nanocrystalline diamond films [7] [23]. A region with negative magnetoresistance is found when decreasing the magnetic field. Superconducting behaviour is found at sufficiently weak magnetic fields, a qualitative similar behaviour has been reported in other granular systems [40] [53] [54].

The physics of granular metallic systems with weak intergranular coupling has been summarized recently by Beloborodov et al. [44]. Hereby, the negative magnetoresistance of a granular metal can be explained by the suppression of the density of states (DOS) due to superconducting fluctuations [55]. As layed out in Ref. [56], the theory of superconducting fluctuations near the transition into the superconduting state was developed long ago. The formation of a new transport channel via nonequilibrium Cooper pairs leads to the so-called Aslamazov-Larkin contribution $\delta \sigma_{A L}$ ) [57]. Coherent scattering of electrons forming a Cooper pair on impurities gives rise to the Maki-Thompson contribution $\delta \sigma_{M T}$ [58] [59]. Both of these corrections enhance the conductivity, therefore leading to a positive magnetoresistance. However, a fluctuational gap in the oneelectron spectrum is opening up due to the formation of nonequilibrium Cooper pairs [60]. This suppression of the density of states has been shown to lead to a correction of the conductivity $\delta \sigma_{D O S}$ [55]. Beloborodov et al. has shown that in a granular systems the DOS correction plays a very important role [55] [61], whereas in conventional superconductors $\delta \sigma_{A L}$ and $\delta \sigma_{M T}$ are more important than $\delta \sigma_{D O S}$. This contribution to the conductivity $\delta \sigma_{D O S}$ is negative. Its absolute value decreases with increasing magnetic field, thus leading to the negative magnetoresistance which is seen for sample Bus4000 in Fig. 6 (b).

One should keep in mind that for sample Bus4000 $\mathrm{g}$ is close to one, approaching the quantum resistance $R_{0}=h /(2 e)^{2} \approx 6.4 k \Omega$ for which a superconductorinsulator transition is expected. In this case, similar behaviours of the magnetoresistance have been reported [62] [63] [64]. The origin of such a transition is still under debate. Some theories claim that it may be understood in terms of Cooper-pair scattering out of the superconducting condensate into a Bose-glass [65] [66], others claim that a percolation description of the superconductor-insulator transition is more adequate [67]. Hereby global superconductivity is destroyed by breaking up the weak links between the superconducting grains (Josephson junctions) at low magnetic fields. At strong magnetic fields even the superconductivity in the grains is broken. The negative magnetoresistance is due to the increasing number of accessible states for normal transport in the phase space [67]. a)

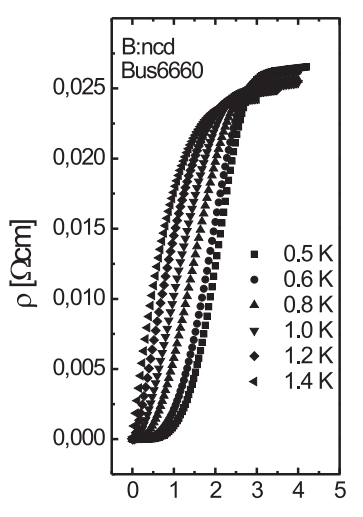

C)

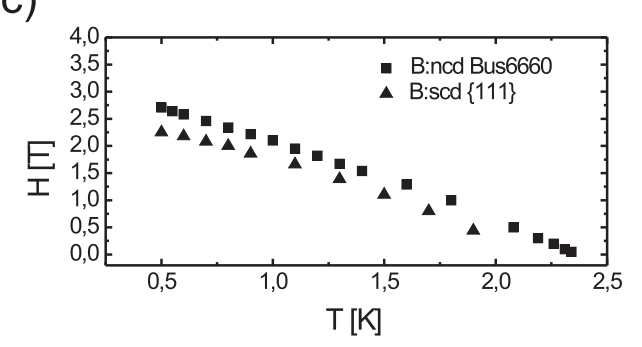

Figure 7 (a) Magnetoresistance for sample Bus6660 at several constant temperatures; (b) similar to results obtained on equivalent heavily boron-doped single crystal diamond films. For sample Bus6660 $g \approx g_{0} \gg 1$ holds and the properties are not very different from those of the bulk superconductor; (c) Phase diagram obtained from temperature sweeps at constant magnetic field and magnetic field sweeps at constant temperature indicate a critical magnetic field $H_{c 2}$ of about $2.5 \mathrm{~T}$ comparable to values obtained in single crystal material.

Fig. 7 (a) shows the magnetoresistance for sample Bus6660 at several constant temperatures. With increasing magnetic field superconductivity is broken, the onset is shifted to lower magnetic field values with increasing temperature. This behaviour is quantitativly similar to results obtained on equivalent heavily boron-doped single crystal diamond films, as shown for a highly boron-doped single crystal $\{111\}$ diamond sample in Fig. 7 (b). Fig. 7 (c) shows the phase diagram $H_{c 2}(T)$ a critical magnetic field of about $2.5 T$ is estimated, similar to single crystal diamond in the same doping range. Moreover, the temperature dependence of the conductivity at low temperatures of highly boron-doped nanocrystalline diamond films (not shown here) can be explained by localization effects and electron-electron interactions typical for disordered metals (see Ref. 50 and references therein), following

$$
\sigma_{T}=\sigma_{0}+A \times T^{1 / 2}+B \times T^{p / 2}
$$

where $\mathrm{p}$ is an index depending on the scattering mechanism [52], and $\sigma_{0}$ the finite conductivity extrapolated to zero 
temperature, similar to single crystal diamond [17]. One possibility is that the intergranular conductance $g$ increases with doping. Therefore, one reaches $g \sim g_{0}$, and these samples should be treated as a homogeneously disordered system which explains the similarities in the electronic transport properties of highly boron-doped nanocrystalline and single crystal diamond films.

4 Conclusion We report on the metal-insulator transition in highly boron-doped nanocrystalline diamond films. Low temperature transport measurements suggest that metallic nanocrystalline diamond follows dependencies for the conductivity typical for a granular system with metallic grains and strong or low intergranular coupling. On the insulating side of the transition, consisting of connected insulating grains, Efros-Shklovskii-like variable range hopping was observed, while on the metallic side a logarithmic temperature dependence of the conductivity was found. The granular structure influences the superconducting properties and the magnetoresistance in highly boron-doped nanocrystalline diamond films. Tuning the microscopic parameters leads to rich behaviour and similarities to the superconductor-insulator transition are pointed out, explained on the basis of superconducting fluctuations and the importance of granularity.

Acknowledgements An acknowledgement may be placed at the end of the article.

\section{References}

[1] E.A. Ekimov, V.A. Sidorov, E.D. Bauer, N.N. Mel'nik, N.J. Curro, J.D. Thompson, and S.M. Stishov, Nature 428, 542 (2004).

[2] Y. Takano, M. Nagao, L. Sakaguchi, M. Tachiki, T. Hatano, K. Kobayashi, H. Umezawa, and H. Kawarada, Appl. Phys. Lett. 85, 2851 (2004).

[3] K. Winzer, D. Bogdanov, and Ch. Wild, Physcia C 432, 65 (2005).

[4] E. Bustarret, J. Kacmarcik, C. Marcenat, E. Gheeraert, C. Cytermann, J. Marcus, and T. Klein, Phys. Rev. Lett. 93, 237005 (2004)

[5] V.A. Sidorov, E.A. Ekimov, S.M. Stishov, E.D. Bauer, and J.D. Thompson, Phys. Rev. B 71, 060502(R) (2005).

[6] Z.L. Wang, Q. Luo, L.W. Liu, C.Y. Li, H.X. Yang, H.F. Yang, J.J. Li, X.Y. Lu, Z.S. Jin, L. Lu, and C.Z. Gu, Diam. Rel. Mat. 15, 659 (2006)

[7] M. Nesládek, D. Tromson, C. Mer, P. Bergonzo, P. Hubik, and J.J. Mares, Appl. Phys. Lett. 88, 232111 (2006).

[8] E. Bustarret, P. Achatz, B. Sacépé, C. Chapelier, C. Marcenat, L. Ortga, and T. Klein, Phil. Trans. R. Soc. A 366, 267 (2008).

[9] E. Bustarret, phys. stat. sol. (a) 205997 (2008).

[10] G. Baskaran, cond-mat/0404286 (unpublished).

[11] G. Baskaran, Sci. Tech. Adv. Mat. 7, S49 (2006).

[12] J.J. Mares, P. Hubík, M. Nesládek, D. Kindl, and J. Kristofik, Diam. Rel. Mat. 15, 1863 (2006).

[13] J.J. Mares, M. Nesládek, P. Hubík, D. Kindl, and J. Kristofik, Diam. Rel. Mat. 16, 1 (2007).
[14] J.J. Mares, M. Nesládek, P. Hubík, and J. Kristofik, Diam. Rel. Mat. 16, 921 (2007).

[15] J.J. Mares, P. Hubík, J. KristofikM, and Nesládek, Sci. Technol. Adv. Mater. 9, 044101 (2008).

[16] T. Tshepe, C. Kasl, J.F. Prins, and M.J.R. Hoch, Phys. Rev. B 70, 245107 (2004).

[17] T. Klein, P. Achatz, J. Kacmarcik, C. Marcenat, F. Gustafsson, J. Marcus, E. Bustarret, J. Pernot, F. Omnes, Bo E. Sernelius, C. Persson, A. Ferreira da Silva, and C. Cytermann, Phys. Rev. B 75, 165313 (2007).

[18] O.A. Williams, O. Douhéret, M. Daenen, K. Haenen, E. Osawa, and M. Takahashi, Chem. Phys. Lett. 445, 255 (2007).

[19] A.C. Ferrari and J. Robertson, Phys. Rev. B 61, 14095 (2000).

[20] P. Gonon, E. Gheeraert, A. Deneuville, F. Fontaine, L. Abello, and G. Lucazeau, J. Appl. Phys. 78, 7059 (1995).

[21] J.W. Ager, W. Walukiewicz, M. McCluskey, M.A. Plano, and M.I. Landstrass, Appl. Phys. Lett. 66, 616 (1995).

[22] Y. Wang, H. Li, Z. Lin, and K. Feng, Jpn. J. Appl. Phys. 39, 2795 (2000).

[23] E. Bustarret, E. Gheeraert, and K. Watanabe, phys. stat. sol. (a) 199, 9 (2003).

[24] W. Gajewski, P. Achatz, O.A. Williams, K. Haenen, E. Bustarret, M. Stutzmann, and J.A. Garrido, Phys. Rev. B 79, 045206 (2009).

[25] H. Fritzsche and M. Pollak, Hopping and Related Phenomena (World Scientific, Singapore, 1989).

[26] B.I. Shklovskii and A.L. Efros, Electronic Properties of Doped Semiconductors (Springer-Verlag, Berlin, 1988).

[27] A. Miller and E. Abrahams, Phys. Rev. 120, 745 (1960).

[28] N.F. Mott, J. Non-Cryst. Solids 1, 1 (1968).

[29] M. Pollak, Philos. Mag. 23, 519 (1971).

[30] A.L. Efros and B.I. Shklovskii, J. Phys. C 8, L49 (1975).

[31] A.L. Efros and B.I. Shklovskii, J. Phys. Solid State Phys. 9, 2021 (1976).

[32] T. Sato, K. Ohashi, H. Sugai, T. Sumi, K. Haruna, H. Maeta, N. Matsumoto, and H. Otsuka, Phys. Rev. B 61, 12970 (2000).

[33] C.J. Adkin, J. Phys.: Condens. Matter 1, 1253 (1989).

[34] A. T. Collins, The Physics of Diamond (IOS Press, Amsterdam, 1997)

[35] I. Shlimak, M. Kaveh, R. Ussyshbin, V. Ginodman, S. D. Baranovskii, P. Thomas, H. Vaupel, and R. W. van der Heijden, Phys. Rev. Lett. 75, 4764 (1995).

[36] N. F. Mott and M. Kaveh, Adv. Phys. 34, 329 (1985); 34, 367 (1985).

[37] B. Abeles, P. Sheng, M.D. Coutts, and Y. Arie, Adv. Phys. 24, 407 (1975).

[38] T. Chui, G. Deutscher, P. Lindenfeld, and W.L. McLean, Phys. Rev. B 23, 6172 (1981).

[39] R.W. Simon, B.J. Dalrymple, D. Van Vechten, W.W. Fuller, and S.A. Wolf, Phys. Rev. B 36, 1962 (1987).

[40] A. Gerber, A. Milner, G. Deutscher, M. Karpovsky, and A. Gladkikh, Phys. Rev. Lett. 78, 4277 (1997).

[41] D. Yu, C. Wang, B.L. Wehrenberg, and P. Guyot-Sionnest, Phys. Rev. Lett. 92, 216802 (2004).

[42] H.E. Romero and M. Drndic, Phys. Rev. Lett. 95, 156801 (2005).

[43] T.B. Tran, I.S. Beloborodov, X.M. Lin, T.P. Bigioni, V.M. Vinokur, and H.M. Jaeger, Phys. Rev. Lett. 95, 076806 (2005). 
[44] I.S. Beloborodov, A.V. Lopatin, V.M. Vinokur, and K.B. Efetov, Rev. Mod. Phys. 79469 (2007).

[45] M.V. Feigel'man and A.S. Ioselevich, JETP Lett. 81, 227 (2005).

[46] I.S. Beloborodov, K.B. Efetov, A.V. Lopatin, and V.M. Vinokur, Phys. Rev. B 71, 184501 (2005).

[47] J. Zhang and B.I. Shklovskii, Phys. Rev. B 70, 115317 (2004).

[48] A. Gerber, J. Phys.: Condens. Matter 28161 (1990).

[49] C. Radhakrishnan, C.K. Subramaniam, S. Sankaranaranyanan, G.V.S. Rao, and R. Srinivasen, Physica C 167, 53 (1990).

[50] H. Fujimori, S. Mitani, S. Ohnuma, T. Ikeda, T. Shima, and T. Matsumoto, Mater. Sci. Eng. A 181-182, 897 (1994).

[51] L. Rotkina, S. Oh, J.N. Eckstein, and S.V. Rotkin, Phys. Rev. B 72, 233407 (2005).

[52] P.A. Lee and T.V. Ramakrishnan, Rev. Mod. Phys. 57287 (1985).

[53] V.F. Gantmakher, M. Golubkov, J.G.S. Lok, and A. Geim, JETP Lett. 82, 951 (1996).

[54] R. Parthasarathy, X.-M. Lin, K. Elteto, T.F. Rosenbaum, and H.M. Jaeger, Phys. Rev. Lett. 92, 076801 (2004).

[55] I.S. Beloborodov and K.B. Efetov, Phys. Rev. Lett. 82, 3332 (1999).

[56] A.I. Larkin and A. Varlamov, Theory of Fluctuations in Superconductors (Oxford University Press, New York, 2005).

[57] L.G. Aslamazov and A.I. Larkin, Sov. Phys. Solid State 10, 875 (1968).

[58] K. Maki Prog. Theor. Phys. 39, 897 (1968)

[59] R.S. Thompson, Phys. Rev. B 1, 327 (1970).

[60] E. Abrahams, M. Redi, and J. Woo, Phys. Rev. B 1, 208 (1970).

[61] I.S. Beloborodov, K.B. Efetov, and A.I. Larkin, Phys. Rev. B 61, 9145 (2000).

[62] A.F. Hebard and M.A. Paalanen, Phys. Rev. Lett. 65, 927 (1990).

[63] M.A. Paalanen, A.F. Hebard, and R.R. Ruel, Phys. Rev. Lett. 69, 1604 (1992).

[64] N. Mason and A. Kapitulnik, Phys. Rev. Lett. 82, 5341 (1999)

[65] M.P.A. Fisher, Phys. Rev. Lett. 65, 923 (1990)

[66] E.S. Sorensen, M. Wallin, S.M. Girvin, and A.P. Young, Phys. Rev. Lett. 69, 828 (1992)

[67] Y. Dubi, Y. Meir, and Y. Avishai, Phys. Rev. B 71, 125311 (2005). 